\title{
The Rossi X-Ray Timing Explorer: Capabilities, Achievements and Aims
}

\author{
J. H. Swank \\ Code 662 Goddard Space Flight Center, Greenbelt, MD 20740, U. S. A.
}

\begin{abstract}
The prime scientific objectives of the Rossi X-Ray Timing Explorer (RXTE) were the study of astrophysical compact objects: black holes (galactic and extragalactic), many types of neutron stars, and accreting white dwarfs. RXTE was successful in achieving its original observing objectives of large area and high time resolution observations with broadband (2-200 keV) spectra, scheduled flexibly enough to enable observations of targets of opportunity on any timescale greater than a few hours. These capabilities enabled qualitatively new discoveries about dynamical timescale phenomena related to neutron stars and black holes, phenomena which probe basic physics in the most extreme environments of gravity, density, and magnetic fields. RXTE has extended its lifetime by applying the proportional counter area selectively and maintains schedule flexibility by making use of the distribution of targets around the sky. Proposed future observations emphasize opportunity to discover and study additional millisecond pulsars, pursue the high frequency quasi-periodic oscillations in black hole transients, and connect high frequency phenomena with longer term characteristics. RXTE will continue to strongly support, for both galactic and extragalactic targets, combining RXTE observations with other wavelengths (from IR to $\mathrm{TeV}$ ) or with other capabilities, such as high spectral resolution.
\end{abstract}

Key words:

\section{Introduction}

$R X T E$ evolved from studies of cosmic X-ray sources with many missions. With $U H U R U$ compact objects were found to be in binary systems and pulsars were found with periods identifying them as neutron stars. Fast irregular variability characterized Cyg X-1. With $A N S$, OSO-8, and $S A S-3$, thermonuclear bursts were identified on neutron stars in low-mass X-ray binaries (LMXB), With $H E A O-1$ jitter in pulsar frequencies could be measured on day time-scales.

'Preprint submitted to Elsevier Science 
EXOSAT saw that the LMXB like Sco X-1 and GX 5-1 had previously unseen internal structure in the emission regions and one could think about measuring clumps in accretion disks as they circulated. The possibility of seeing matter in the innermost stable orbits around black holes and neutron stars promised avenues to actually get information about the laboratories of the densest matter that nature presents, neutron stars and black holes. The goal of accomplishing this dictated the characteristics of RXTE.

\section{Capabilities: Mission, Spacecraft, and Instruments}

$R X T E$ was launched into a circular $580 \mathrm{~km}$ low-earth orbit, which has decayed during 10 years (and one solar maximum) to about $490 \mathrm{~km}$. The inclination of about $23^{\circ}$ is the lowest that could be achieved by its launch latitude and the energy available from the Delta 2 launch rocket which carried it on December 30, 1995. Data is telemetered to the TDRSS and thence to White Sands.

The spacecraft was built by Goddard Space Flight Center and designed to satisfy the requirements of the RXTE instruments. Hence the star trackers can for the most part update the attitude during the detector observations. Initially RXTE could observe in any direction, while still being accessible to commands and able to telemeter data. But the redundancy of the high gain antennas served to rescue the mission when one of the transponders failed in September 1999. The spacecraft is 3-axis stabilized and holds the bore-sight attitude to about 10 arc seconds of the desired pointing position, and has sufficient mass that the response to motions of the ASM and HEXTE detectors are acceptable. The PCA collimator fields of view are slightly rounded by $\approx$ $0.1^{\circ}$, due to dispersion of the collimating tube directions, and the bore-sights of the detectors are offset from each other by similar amounts. The spacecraft and components are shown in the diagram in Figure 1.

The Proportional Counter Array (PCA) comprises 5 detectors scaled up from the xenon gas detectors that flew on $H E A O-1$. Each has an area of $1250 \mathrm{~cm}^{2}$ for a total of $6250 \mathrm{~cm}^{2}$. The good event cells are shielded on 4 sides by anticoincidence and the long narrow detector shape mitigates against particles traveling parallel to the wires. The $1 \mathrm{~atm}$. pressure, the depth of the counters, the cell sizes, and the construction techniques are those of the detectors flown by the Goddard X-Ray Astrophysics group on rocket flights, balloons, $O S O-8$ and $H E A O-1$. The energy range is $2-60 \mathrm{keV}$. The field of view is $1.2^{\circ}$ hexagonal, small enough to avoid most source confusion except in the center of our Galaxy, and to reduce the background due to the X-ray background that on this spatial scale is "diffuse". The data from all five detectors of the PCA are digitized and fed to the Experiment Data System built by MIT. This processes some of the data, as the storage and transmission resources did not 


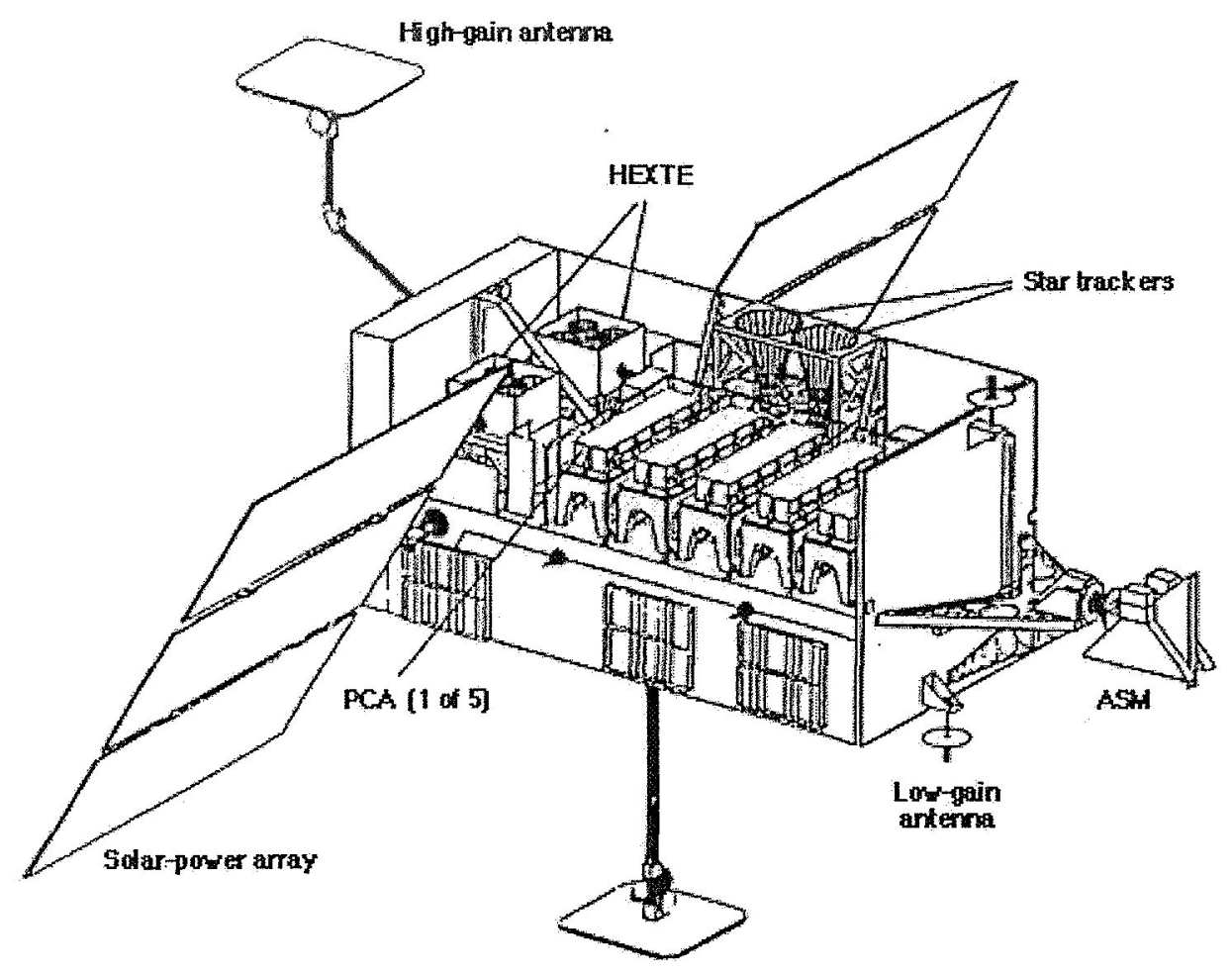

Fig. 1. Diagram of the RXTE Spacecraft. The cutaway drawing shows the simple spacecraft platform, the five proportional counter units, the two HEXTE clusters, each of four detectors, the three Scanning Shadow Counters on the boom extending from the spacecraft, the two star trackers, solar arrays and two high gain antennas.

allow all the data to be brought to the ground. Rates of all types of events are sent down, those identified as due to particles as well as good X-ray events; they are used in analysis to calibrate the "background" of counts in the detectors that escape being flagged and excluded as particle events. The total background counting rate is about 100 counts s$^{-1}(2-60 \mathrm{keV})$. The $2-10 \mathrm{keV}$ background counting rate due to both $\mathrm{X}$-rays and particles is equivalent to an approximately $2 \mathrm{mCrab}$ source. The achieved resolution of the detectors is near the limit of about $18 \%$ at $6 \mathrm{keV}$ for xenon proportional counters. Jahoda et al. (2006) gives a detailed description of the PCA and its calibration.

The High Energy X-ray Timing Experiment (HEXTE) is the RXTE way to increase the high energy area above that of the PCA. Two sets of $4 \mathrm{NaI} / \mathrm{CsI}$ Phoswich detectors provide $1600 \mathrm{~cm}^{2}$ of area $15-200 \mathrm{keV}$ (Rothschild et al., 1998). One of these detectors has provided rates, but not spectra, because of a fault in the Analog to Digital Converter that appeared after launch. For most of the mission these were alternately canted on and off source to allow background to be obtained from $1.5-3^{\circ}$ of the source and within 16$32 \mathrm{~s}$ of on-source. A particle monitor keeps track of passage of the South American Anomaly and controls the voltage on the HEXTE detectors. For some observations of pulsars it has been deemed more valuable to have higher 
area on source and to avoid temporal aliases, by having all detectors stare at the source. After ten years the mechanical modulation of one of the clusters has failed and it has since been pointed directly on-source. The HEXTE detectors have a resolution of $18 \%$ at $60 \mathrm{keV}$.

The All Sky Monitor has been much appreciated by many observers at all wavelengths. The 3 "Scanning Shadow Cameras" which view the sky through coded masks with one dimensional position sensitive anodes step across the sky in 6 degree and $100 \mathrm{~s}$ increments (Levine et al., 1996). The snapshot samples build up light curves of over 200 sources above a few mCrab in intensity. The sensitivity limit depends on source confusion in the region of sky around a source and ranges from a few $\mathrm{mCrab}$ for isolated blazars on a day time scale to $30 \mathrm{Crab}$ for X-ray binaries in our Galactic Bulge. The light curves are posted on line by MIT and Goddard. If the data cannot be fit with sources in the known catalog of sources, a new source is the probable cause and the data can be fit for its position. Accuracies of a few arc minutes are achieved.

It has been possible during the decade of $R X T E$ operations to accept requests for observations on short notice based on information from other observations or from analysis of the $R X T E$ data itself. A turn-around of 8 hours has been our goal. How fast it can be done depends on the luck of the pointing directions at the time compared to the target requested. It can be within an hour, or in unlucky circumstances it might be 3 days. $R X T E$ calculates its slew motions on-board, but it cannot autonomously decide to slew.

\section{Achievements}

\subsection{Black Holes}

Black holes are certainly among the front-runners for being one of the most important types of astronomical objects. Several of the original scientific objectives of $R X T E$ pertained to black holes. An ultimate goal is to confirm the relations between the parameters in General Relativity (GR) that describe the properties of a black hole. The masses of some of the stellar black holes are determined dynamically from the Newtonian motion of a binary companion. There are other possible measures of the mass, for examples, the time-scales of variability, specific quasi-periodic oscillations, and spectra. The angular momentum of the black hole had begun to be discussed before the launch of $R X T E$, in connection with the discovery of the radio jets ejected at the beginning of the 1994 outbursts of GRO J1650-40 and GRS J1915+105. Consideration of the interpretation of the set of QPO from low frequencies (a few Hertz) to the high frequency $(100-500 \mathrm{~Hz})$ and the spectroscopy of black hole 


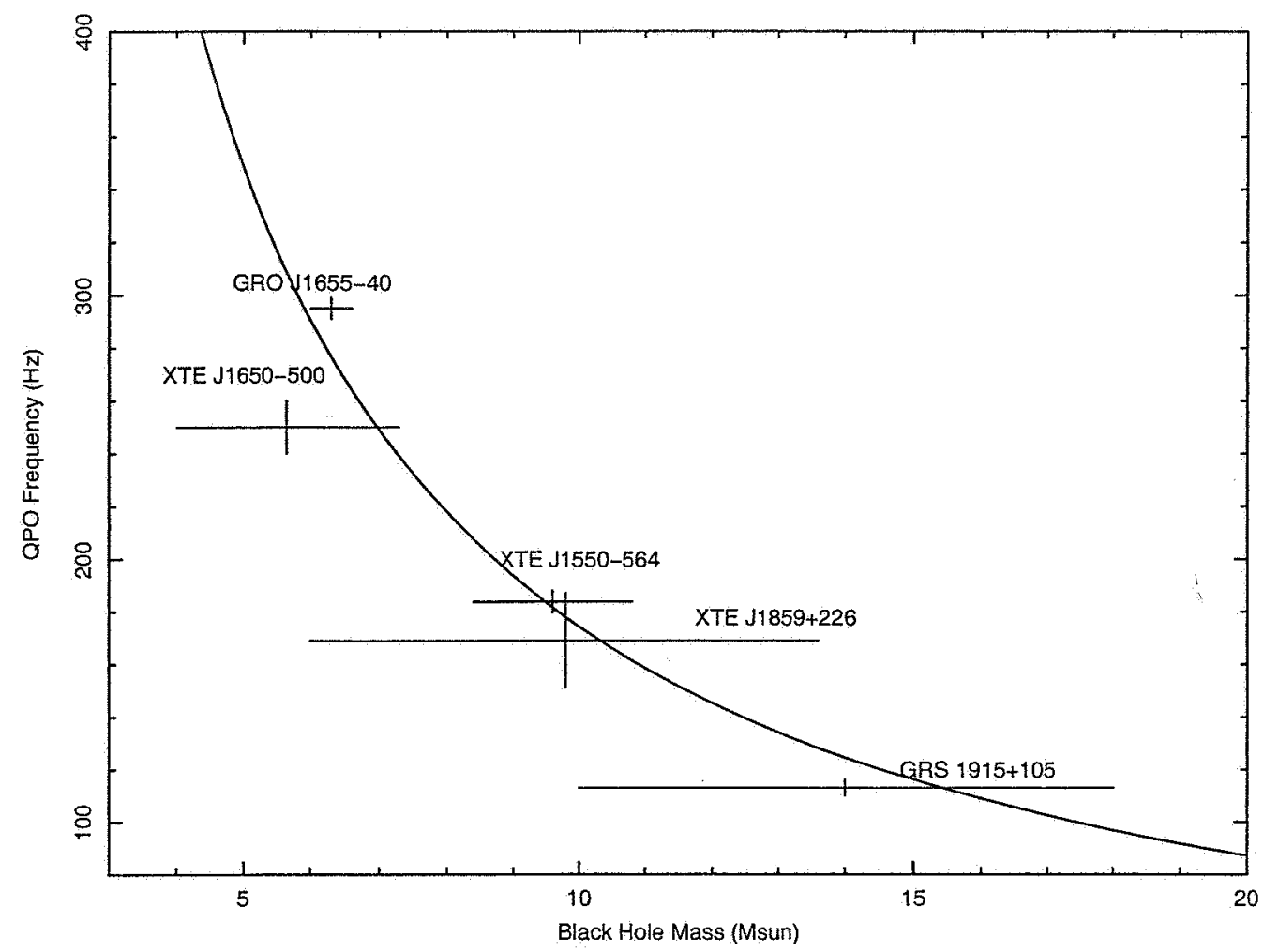

Fig. 2. HFQPO for known Black Hole Mass. Adopted from McClintock \& Remillard (2003). The frequencies for the sources in blue are the lower of a pair that has a 3:2 ratio relationship. Those for the points in red are for the only HFQPO for those sources. Mass measurements are still lacking for H1743-322 and 4U 1630-47 among the $7 \mathrm{BHCs}$ for which frequencies above $100 \mathrm{~Hz}$ are detected. Accurate mass measurements are important.

candidates has shown that they both are handles on the angular momenta of black holes (McClintock \& Remillard, 2003).

In GR there should be an "ergosphere" around black holes of a given mass $M$ and dimensionless angular momentum $a$. Particle and photon orbits around the black hole are only to be found outside of the ergosphere, so that the measurement of an inner radius of an accretion disk determines a locus of $M$ and $a$. Multiple measurements from different techniques can test the consistency of GR. RXTE measurements have inspired theoretical work needed to understand the relation between observables and the physical quantities.

RXTE observations have discovered 13 of 43 binary black holes identified in the Galaxy. Whether there are dynamical masses depends on optical identification and spectroscopy during quiescence. There are 12 now with masses determined to an accuracy of $10 \%$. The high frequency quasi-periodic oscillations have been seen in the x-ray flux of 7 sources, 5 of which have mass measurements. The high frequency QPO has a low amplitude, less than a few per cent. It has been observed only in very transient states of the sources; the source 
has high luminosity and is in an apparently unstable state with both high energy power law emission and optically thick disk emission. This state is sometimes called the "Very High State", sometimes the "Steep Power Law" state. The 7 sources which have shown these QPO are (with parenthetical mass estimates in $\left.M_{\odot}\right)$, XTE J1550-564(8.4-10.8), 4U 1630-47, XTE J1650-500 (4.0-7.3) GRO J1655-40 (6.0-6.6), XTE J1859+226(7.6-12.0), H1743-322, and GRO J1915+105 (10.0-18.0). Five of them have pairs of QPO, ranging from $50 \mathrm{~Hz}$ to $450 \mathrm{~Hz}$.The presence of pairs of QPO have inspired several comparisons to theoretical models.

There is not space to describe here the evidence that has been found in many observations of the connections between accretion and jet ejection from black holes, or the similarities between stellar and super-massive black holes, which imply the timing characteristics scale inversely with black hole mass and should help in understand jet production in both kinds of systems.

\subsection{Neutron Stars}

Close to and inside neutron stars is arguably as important a locus of strong gravity as near black holes. Gravity is strong, yet we believe GR is well behaved everywhere, so the situation can be approached from the physics that we believe we know. The realms of macroscopic nuclear density and intense magnetic fields are not elsewhere (after the Big Bang) available for study. We have the good fortune that we have pulsed fluxes, surface thermal spectra, absorption and emission lines, that we basically understand, to enlarge our understanding of the interiors of neutron stars, the behavior of matter in strong magnetic fields and strong gravity, and the evolution of the systems.

The interior physics of neutron stars determines the mass, radius, and moment of inertia and there is a fairly unique correspondence between input physics and the locus of possible variables. Hence there is great interest in methods of determining these properties of neutron stars. They enter into several different kinds of X-ray measurements. Thermonuclear X-ray bursts have spectra close to optically thick black body emission and peak luminosities that should be related to the Eddington-limited flux for the kind of material that would have been accreted. These give a handle on mass and radius of the neutron star, with uncertainties coming from uncertainties in the source distances and the model of the distribution of radiating material on the neutron star. RXTE discoveries of kilohertz oscillations from the accreting neutron stars in low-mass $\mathrm{X}$-ray binaries gives a completely different approach, if the highest frequency observed can be taken to be from the Kepler orbit of material orbiting at the inner-most stable circular orbit (ISCO). Then $M=2200 / \nu$ except for corrections due to spin (Miller, 2004, e.g.). The spins are now known for 21 LMXB, 
including 7 accreting millisecond pulsars; the maximum spin frequency is so far the $619 \mathrm{~Hz}$ of $4 \mathrm{U}$ 1608-522. Probably not until details of the model are worked out and shown to be in accordance with the extensive well-defined phenomenology, will this be accepted as definitive. But it promises to be a very powerful constraint on mass and radius. The millisecond pulsations that

the RXTE PCA has discovered provide still another approach. Whether the hot spot is persistently present or is fleeting during a thermonuclear flash burst, its appearance as a function of phase and energy is affected by a photon's geodesic as it escapes the gravitational field of the neutron star and the Doppler shifts of the hot spot rotating on the neutron star. The different techniques (Poutanen \& Gierliński, 2003; Bhattacharyya et al., 2005) give answers in a reasonable mass and radius range for neutron stars and begin to constrain the equation of state.

\section{Summary}

Many results about strong gravity, high density, and strong magnetic field are being obtained and published which are of interest to studies of compact objects. The pre-launch objectives were phrased generally and guest observers have been able to productively follow up on discoveries. The detailed emphasis of the research is a little different from the original rationale for the mission. Pre-launch we could not be sure that the very interesting timing signals in the kilohertz range would actually be found with amplitudes that would allow extensive study. We proposed to look for them because they would be so interesting if found, but we did not anticipate the detail. We did know that pulsars exhibit strong frequency fluctuations which theoretically offer a handle on the interior structure of neutron stars. RXTE observations have found glitches in pulsars and oscillation frequencies in magnetars that start to make. real progress on these questions as well, though the complexity of the pulse behavior of accreting pulsars has made it difficult to draw conclusions from their frequency variations. But the high time resolution results have raised many questions that we hope to continue to gather evidence to answer.

It may take resolving weaker features in the power density spectra to distinguish between alternative models. We are still at the stage of establishing how the systems work. When that is secure the phenomena could be used to determine the range of masses and angular momenta evolved in neutron star LMXB and in black hole binaries and to look for more detailed behavior of space time and matter in strong gravity, for example. It may require new missions with more powerful capabilities to do this (Lamb, 2004, e.g.).

RXTE has found 7 millisecond accretion pulsars and 7 black hole transients with HFQPO. A few more sources of both types, which we can reasonably ex- 
pect to discover in a few more years, can make a significant difference to our understanding. At the moment there is one pulsar with a kilohertz frequency difference equal to the pulse frequency and one for which it is half the pulse frequency. A theory to explain this dichotomy requires confirmation of its predictions. At the moment four black hole candidates have two frequencies in a ratio of $3: 2$ and in fact GRS $1915+105$ has two sets of frequencies with approximately this ratio. Another black hole candidate exhibiting the phenomena could offer definitive information. $R X T E$ observations of blazars in coordination with high energy $\gamma$-ray measurements have been very interesting, but so far, only a few sources have been accessible to the $\mathrm{TeV}$ observatories. The number of targets accessible to $\mathrm{TeV}$ measurements is increasing with completion of those observatories. The anticipated high-energy $\gamma$-ray observatory GLAST (and its precursor $A G I L E$ ) will increase by an order of magnitude the number of sources which can be monitored simultaneously.

We hope that the launch of $A S T R O S A T$ will allow astrophysics to continue beyond $R X T E$ to use timing to investigate compact objects, and to add the tools of its unique combination of capabilities.

\section{References}

Bhattacharyya, S., Strohmayer, T. E., Miller, M. C., \& Markwardt, C. B., Constraints on Neutron Star Parameters from Burst Oscillation Light Curves of the Accreting Millisecond Pulsar XTE J1814-338, 2005, ApJ, 619, 483-491

Jahoda, K., Markwardt, C. B., Radeva, Y., Rots, A. H., Stark, M. J., Swank, J. H., Strohmayer, T. E., \& Zhang, W., Calibration of the Rossi X-Ray Timing Explorer Proportional Counter Array, 2006, ApJS, 163-401

Lamb, F. K., Scientific Challenges for a New X-ray Timing Mission, 2004, AIP Conf. Proc. 714: X-ray Timing 2003: Rossi and Beyond, 714, 3-12

Levine, A. M.; Bradt, H., Cui, W., Jernigan, J. G., Morgan, E. H., Remillard, R., Shirey, R. E., \& Smith, D. A., First Results from the All-Sky Monitor on the Rossi X-Ray Timing Explorer, 1996, ApJ, 469, L33-36

McClintock, J. E., \& Remillard, R. A., Black Hole Binaries, 2003, in Compact Stellar X-Ray Sources, eds. W. H. G. Lewin \& M. van der Klis, Cambridge University Press, in press, astro-ph/0306213

Miller, M. C., Interpreting QPOs from Accreting Neutron Stars 2004, AIP Conf. Proc. 714: X-ray Timing 2003: Rossi and Beyond, 714, 365-370

Poutanen, J., \& Gierliński, M., On the nature of the X-ray emission from the accreting millisecond pulsar SAX J1808.4-3658, 2003, MNRAS, 343, 13011311

Rothschild, R. E., et al., In-Flight Performance of the High-Energy X-Ray Timing Experiment on the Rossi X-Ray Timing Explorer, 1998, ApJ, 496538 